\title{
Introducción al análisis del trabajo y de la vida cotidiana
}

\author{
Faustino M iguélez \\ Teresa Torns \\ Universitat Autònoma de Barcelona. D epartament de Sociologia \\ 08193 Bellaterra (Barcelona). Spain
}

\section{Resumen}

El artículo plantea la introducción a la investigación y, en este sentido, a los artículos posteriores. A parecen las preocupaciones y la problemática que están en el origen de la investigación, es decir, la necesidad de superar los análisis sectoriales en los campos de la producción, reproducción y tiempo libre para dar precisamente cuenta de hechos sociales vinculados a la relación entre ellos, aparte de ofrecer un visión de cada uno de ellos. Establece los objetivos de la investigación y los marcos teóricos de referencia, concretamente la perspectiva de la vida cotidiana y las teorías que vinculan producción y reproducción, así como las fases, niveles de tratamiento, las grandes orientaciones metodologicas y las técnicas utilizadas.

Este artículo es parte de una investigación cuyos resultados aparecen en este mismo número de PAPERS. El artículo tiene autonomía y entidad por sí mismo pero la lectura del resto de los artículos de este número facilitará su contextualización.

Palabras clave: producción, reproducción, tiempo libre, vida cotidiana.

\section{Abstract}

This article presents the introduction to the research and to the later articles. It deals with the concerns and the problems that form the basis for the research, that is to say, the need to go beyond sectorial analysis in the fields of production, reproduction and free time in order to account for social facts arising from the relation between them and to offer a view of each one. It establishes the objectives of the research and the theoretical frameworks of reference, specifically the perspective of daily life and the theories that link production and reproduction as well as the phases, levels of processing, major methodological guidelines and techniques used.

This paper is part of a research work whose results are in this PAPERS volume. The paper is autonomous but the reading of the other papers of this volum can help to contextualize it.

Key words: production, reproduction, free time, everyday life. 


\section{Sumario}

1. Origen de la investigación

2. Los cambios en el trabajo

3. Cambios en el ámbito del trabajo reproductivo

4. El tiempo libre
5. Objetivos de la investigación

6. Marco teórico de la investigación

7. Los niveles de la aproximación metodológica a los ámbitos y a su interacción

Bibliografía

\section{O rigen de la investigación}

En el equipo que desarrolla el proyecto, que está en la base de estos artículos, han coincidido profesores y profesoras y/o investigadores e investigadoras que tienen intereses específicos en cada uno de los campos de investigación como son el trabajo, en su doble acepción productiva y reproductiva, y el uso del tiempo, y que participan de una común insatisfacción en cuanto a los resultados de las investigaciones que tienden a no salir de su ámbito, potenciando separaciones artificiales que no existen en la vida D e ahí se ha derivado el deseo de buscar una nueva perspectiva investigadora más interactiva entre los campos enumerados, que hemos acabado denominando «vida cotidiana».

Sin duda el campo que más se ha investigado es el del trabajo. $\mathrm{H}$ ay muchos y diversos estudios realizados en los últimos años sobre el mercado de trabajo, la descentralización, el trabajo sumergido, las relaciones laborales, la organización del trabajo, etc. Precisamente una de las más sólidas conclusiones de estos estudios parece mostrar que lo que circunscribimos y definimos como trabajo está fuertemente marcado por factores externos al trabajo llamado productivo. Dichos factores, que podríamos Ilamar socioculturales, se extienden por ejemplo al ámbito familiar, al colectivo, al individual, por lo que evidentemente no se sitúan directamente en el campo de la sociología del trabajo. Estos factores externos, sin embargo, son muy poco tenidos en cuenta como factores explicativos del trabajo mismo, de la cotidianidad del trabajo.

En un segundo ámbito, el del trabajo reproductivo, se ha investigado sobre la situación social de las mujeres, particularmente en el trabajo de mercado y en el trabajo doméstico. A raíz de tales estudios se ha ido percibiendo cada vez más la necesidad de estudiar el trabajo productivo y reproductivo al mismo tiempo, no sólo en términos de cal endario, sino primordialmente en términos teóricos; es decir, se trata de explicar ambos tipos de actividades conjuntamente, de entender que el trabajo no es sólo el que se desarrolla en el mercado.

En el tercer ámbito, el del tiempo libre, los estudios se han centrado en actitudes y prácticas ante diversas esferas de la vida privada, familiar, en las formas de vida de determinados colectivos, en el uso del tiempo, particularmente del tiempo libre. También aquí las limitaciones provenían por ejemplo de que el tiempo libre no ha sido considerado suficientemente vinculado a otras actividades. 
En suma, una limitación de muchas de estas investigaciones, que no las invalida pero sí las deja alicortas, es que lo que la gente percibe, se representa y hace en un ámbito concreto de la vida social está íntimamente marcado por lo que percibe, se representa o hace en los otros ámbitos. Este nexo no sería captado por una investigación que se quedara limitada a un solo ámbito. ¿H abría alguna posibilidad de tener en cuenta esta implicación mutua en la investigación social sin caer en la pretensión de dejar de lado o minusvalorar otras aproximaciones?

El esfuerzo del equipo en la preparación del proyecto y en la primera fase de la investigación se ha centrado precisamente en responder a esa pregunta, elaborando una línea común de investigación a estos tres campos desde un nuevo punto de vista; esto es, desde una metodología pertinente, tanto en los aspectos teóricos como en los métodos y técnicas. El marco y modelo que nos ha parecido más adecuado para dicho tratamiento ha sido el que denominaremos genéricamente de la «vida cotidiana». En la propuesta que se desarrolla, la vida cotidiana se entiende precisamente como la articulación y organización concreta que la gente realiza entre los diversos ámbitos de su vida social, tanto en términos de la práctica y/o actividad (perspectiva más objetivista) como en términos de percepciones, estados y/o contenidos intencionales y actitudes (perspectiva más simbólica).

Investigar sobre la vida cotidiana en términos globales, es decir, tomando la totalidad de los ámbitos sociales, habría resultado no sólo complejo y de difícil tratamiento, sino también extraordinariamente largo, pensando sobre todo en el corto lapso de tiempo de que disponíamos. Por ello creímos conveniente, aprovechando nuestra experiencia investigadora precedente y tratando de mantener la exigencia de interrelación entre diversas esferas de la vida social, centrarnos solamente en tres ámbitos que, por razones que iremos señalando, nos parecen hoy claves: el trabajo del que se obtienen los recursos para vivir - que llamaremos «ámbito del trabajo productivo»- , las tareas domésticas y del cuidado de la casa y de la familia, que llamaremos «ámbito reproductivo», y el ámbito de las actividades consideradas de tiempo libre. Antes de entrar más a fondo en el desarrollo de los objetivos de la investigación conviene que veamos cuál es el «estado de la cuestión» con respecto a estas tres áreas.

\section{Los cambios en el trabajo}

A lo largo de las últimas décadas los países avanzados están asistiendo a una profunda transformación de las formas de trabajo, transformación que no parece exagerado pensar que tendrá y está teniendo ya una influencia importante en las formas de vida.

El trabajo industrial está siendo sustituido por trabajo de servicios (sean éstos personales 0 a las empresas). Pero además, y con mayor intensidad si cabe, hay que señalar que el trabajo directo (que se traduce en producción de bienes) se reduce y crece el trabajo indirecto. En nuestro país existe ya alguna 
investigación que pone de manifiesto la existencia de ambos fenómenos (C astells y otros, 1986). Los cambios son profundos igualmente en la estuctura ocupacional: se desarrollan las ocupaciones técnico-profesionales, administrativas y el trabajo en los servicios, mientras que decrecen las ocupaciones en la industria y en la agricultura.

Se dibuja un nuevo tipo de división del trabajo que va más allá de la que separa trabajo manual de trabajo intelectual: el trabajo muy cualificado, con iniciativa y con sentido que se desarrolla en ciertas unidades productivas de bienes y servicios, en ciertas ocupaciones (H . Kern, M. Schumann, 1988), frente al trabajo descualificado (aunque no sea de tipo manual), con poca iniciativa y con poco sentido, que parece alimentar los niveles más bajos del sistema. Ambos tipos de trabajo van creciendo fuertemente, pero a mayor ritmo el segundo que el primero, por lo menos si a nuestro país nos referimos.

Sin embargo, en todos estos aspectos, los estudios han podido ir por ahora poco más allá del análisis de los datos estadísticos que los censos o la Encuesta de Población Activa nos ofrecen de la actividad o la ocupación según sectores o por estructura ocupacional. No se ha entrado a fondo en el contenido de estos nuevos trabajos ni, menos aún, en las consecuencias sociales y personales que tienen, tanto en la vida activa en sí como en la vida cotidiana para quienes los realizan.

Por supuesto que algunos aspectos del «nuevo trabajo» están siendo analizados, al menos parcialmente, más allá de los datos estadísticos de base. Por ejemplo, se ha avanzado en el análisis del significado social del trabajo sumergido (M inisterio de Economía, 1985; Sanchís, 1984; Alós, M iguélez, Recio, 1988b, 1989). Diversos organismos, públicos y privados, han iniciado estudios sobre la caracterización de los empleos de «contratación especial», por ejemplo (M TSS, varios años, Enquesta M etropolitana, 1985, 1990). La posibilidad de introducir algún tipo de preguntas, tanto en el censo y padrón como en la Encuesta de Población Activa, sobre las características internas del tra bajo, abrirían nuevas e interesantes perspectivas que sería necesario potenciar.

Estos avances de los últimos años están exigiendo otras investigaciones decididamente centradas sobre la caracterización interna del trabajo, sobre sus transformaciones, sobre el significado que hoy tiene el trabajo para los diversos grupos de personas. Esto es importante desde dos puntos de vista: desde un punto de vista teórico-general, conviene conocer mucho más lo que hoy significa el trabajo en la vida y en el actuar de las personas, por cuanto sólo así podemos entender más a fondo, tanto el presente como el próximo futuro de nuestra sociedad; desde un punto de vista más práctico-político, conviene ana lizar a fondo el posible diverso significado del trabajo para los distintos colectivos sociales, si se quieren realizar políticas económicas y sociales adecuadas en cada caso y no sólo políticas genéricas.

También se han realizado al gunos estudios sobre los factores que están condicionando algunos de los más importantes cambios laborales. La innovación tecnológica en la transformación del trabajo es un tema que está siendo estudiado desde diferentes puntos de vista; por ejemplo, en lo que se refiere a la 
relación entre innovación tecnológica y destrucción o creación de empleo (C astells y otros, 1986), o la relación entre cualificación e innovación tecnológica (H oms, 1988; Lope, 1995), o la que tiene lugar entre nuevas tecnologías y pequeña empresa (C astillo, 1988; M iguélez, López, 1988a; M artín Artiles, 1995).

Por el contrario, las estrategias de los actores han sido menos estudiadas, sean éstos actores individuales (los empresarios, los trabajadores), colectivos (asociaciones empresariales, sindicatos) o institucionales (la Administración a diferentes niveles), aunque no podemos decir que no se hayan hecho al gunos esfuerzos al respecto. Por ejemplo, las estrategias sindicales están siendo estudiadas por algunos equipos en la actual idad, al gunos estudios sobre el empresariado han ido en la misma dirección, aunque no tan recientemente ${ }^{1}$.

Por supuesto, se está investigando sobre la relación entre trabajo y vida cotidiana fuera del trabajo, aunque en menor medida. Se ha estudiado la configuración del uso del tiempo (Enquesta M etropolitana de Barcelona, 1986; M inisterio de Economía y $\mathrm{H}$ acienda, 1985). Se ha profundizado mucho más en la relación trabajo-situación económica, particularmente en el tema de lo que se ha denominado la «nueva pobreza» (D ocumentación Social, 1984; M iguélez, López, 1896b). Pero no se ha investigado en la conexión entre el nuevo significado y las diversidades del trabajo y los cambios globales en la vida cotidiana, sobre todo en el intento de ver cómo se relacionan las estrategias y los actores, en lo que podríamos considerar el nivel dinámico del tema.

Todas estas consideraciones sobre el estado de la cuestión sugieren una serie de reflexiones que constituyen la «justificación», tanto teórica como práctica, de la propuesta de investigación que aquí se desarrolla:

- La primera es que cabe investigar mucho más en lo que se refiere a la evolución interna del trabajo, teniendo en cuenta la influencia de muy diversos factores: económicos (el mercado, la tecnología), sociales (nuevas estrategias de los individuos y de los actores sociales), culturales (nuevos significados del trabajar y del no trabajar) y políticos (papel del Estado y de la sociedad en la configuración del trabajo y de sus significados).

- La segunda es que conviene saber qué personas, por qué motivos y en qué circunstancias ocupan unos u otros puestos de trabajo. No todos los trabajos son iguales: al gunos tienen más que nunca que ver - ésta es nuestra hipótesis- con el poder, la influencia, el control de los valores en alza y con los recursos monetarios que dan una buena posición; otros están en la situación totalmente opuesta; otros, en fin, en posiciones intermedias.

1. Véase en este sentido una investigación sobre el sindicalismo dirigida por S. Aguilar, en la que participan, entre otros, Andreu Lope, Pilar Carrasquer y Albert Recio, que ha producido ya varios volúmenes entre los que destaca el resumen general titulado Els contextos de l'acció sindical.

Respecto a las investigaciones sobre el empresariado, podemos remitir, entre otros, al número monográfico de la Revista Papeles de Economía Española, núm. 22, 1985. 
Q ueremos decir con esto que el tipo de trabajo que se hace tiene bastante que ver con la manera en que aún hoy se configura la vida cotidiana de las personas, dentro del trabajo mismo en primer lugar y fuera del trabajo después.

- La tercera es que resulta muy importante identificar tanto los grupos «de riesgo» - proclives a caer en trabajos poco deseables- como grupos «de privilegio» (con tendencia a ocupar buenos puestos de trabajo no sólo económicamente hablando, sino también desde un punto de vista cultural). Tal cosa es importante con vistas a políticas de bienestar que se basen no exclusivamente en fórmulas asistenciales, sino también en fórmulas participativas de los ciudadanos en las que el diseño del propio trabajo y de la propia vida cotidiana sean, cada vez más, una prerrogativa de cada persona.

D esde el punto de vista teórico, la novedad de esta investigación está en que ofrece la posibilidad de vincular en un solo proyecto datos estructurales e individuales, de relacionar, sobre la base de un mismo esquema teórico - el entramado producción/reprodución-, datos referidos al trabajo y datos referidos a la vida cotidiana en su globalidad.

\section{Cambios en el ámbito del trabajo reproductivo}

A lo largo de las últimas décadas ha aumentado la reivindicación de igualdad de derechos de la mujer, siendo el primero de ellos el acceso al empleo en igualdad de oportunidades, lo que tiene que comportar una mejor distribución de las tareas reproductivas, dado que la no distribución parece ser un «obstáculo» para el mencionado objetivo. Por supuesto que reivindicación no comporta automáticamente cambios, pero esta nueva sensibilidad está aumentando. Por ejemplo, la Enquesta M etropolitana de Barcelona de 1990 muestra que hay una cierta redistribución del tiempo de trabajo doméstico, aunque en todas las circunstancias la mujer sigue teniendo la carga fundamental de las tareas reproductivas, bien del conjunto familiar, bien de los miembros más desvalidos (niños, ancianos). D e modo que podemos describir la siguiente tipología referida a la actividad de las mujeres:

- las mujeres totalmente dedicadas al trabajo reproductivo;

- las mujeres dedicadas a ambos trabajos, realizando doble jornada, aunque con distribuciones muy diferenciadas;

- las mujeres que sólo trabajan (productivamente) en el mercado.

U na importante corriente de investigadoras está impulsando la necesidad de analizar el trabajo de las mujeres considerando, al mismo tiempo, las implicaciones del trabajo productivo y del trabajo reproductivo. Es decir, que no basta ni es válido considerar el trabajo doméstico meramente como un «obstáculo», sino que es necesario considerar que el trabajo es de doble cara; para 
entender cada una de esas caras y ambas a la vez en la implicación de la vida cotidiana de las personas (no sólo de las mujeres, aunque fácticamente sea principalmente de las mujeres), es necesario no perder la vinculación entre uno y otro ámbito.

Asimismo, nuestra investigación va más allá de la conceptualización del trabajo no remunerado como trabajo doméstico, considerando como trabajo reproductivo no sólo el doméstico (infraestructura del hogar), sino también el cuidado de ancianos y ancianas y niños y niñas, y las tareas de mediación entre la familia y la sociedad (gestión en bancos, colegios, administraciones públicas... ) entre otras tareas.

Lo cual nos lleva también al debate sobre el contenido del trabajo reproductivo, tanto en su relación con el trabajo productivo como con el uso del tiempo o la realización personal en el empleo (profesionalización). Pero, sobre todo, en relación con la vida cotidiana.

\section{El tiempo libre}

Los estudios sobre el tiempo libre han tenido escaso desarrollo en nuestro país salvo algunos aspectos, por ejemplo, relacionados con la contabilidad del empleo del tiempo a él dedicado. Sin embargo, la problemática social vinculada al tiempo libre es de suma importancia, no sólo por el creciente volumen temporal que ocupa - con o sin prácticas - sino también por el lugar preponderante que tiene en el imaginario colectivo de la gente. Si la importancia es grande su tratamiento no es sencillo, debido, entre otras cosas, a las múltiples causas por las que la gente accede o se encuentra con el tiempo libre, por la variabilidad de las prácticas que lo cubren - 0 la ausencia de las mismas- , por los distintos contenidos, actitudes y beneficios que cada uno pretende obtener de dichos momentos. Lo menos que se puede decir es que frente a los otros dos ámbitos, éste es el que menor grado de homogeneidad conlleva, al menos en primera instancia.

Los análisis sobre tiempo libre realizados en otros horizontes no sólo se centran en la distribución de la disponibilidad de dicho tiempo, sino también en las actividades que contiene, en el significado del tiempo libre y, extensivamente, de todo tiempo social, en las relaciones del tiempo libre con otros momentos sociales, en la naturaleza de sus agentes 0 actores sociales, en la relación del tiempo libre con el ciclo de vida y, en general, con los diferentes momentos históricos. Así, el tiempo libre pasa a ser no sólo un producto social sino también un revelador social. Las tendencias van desde quienes consideran el tiempo libre, y las actividades a él vinculadas, como supletorio, heterónomo y dependiente de otros ámbitos sociales, por ejemplo la produccción, hasta las que lo consideran autónomo y ponderadamente determinante de la evolución del resto de los ámbitos: incluso la creciente implantación del tiempo libre, mental y fácticamente es visto dentro de la última tendencia, como un signo irreversible de la evolución de la sociedad. 
D e todas maneras y con más fuerza que en los otros ámbitos, nos interesa no sólo el «cuánto» del tiempo libre sino también el «cómo», el «para qué» y con ello el sentido y el imaginario.

\section{0 bjetivos de la investigación}

En nuestro análisis hemos adoptado una posición cautelosa y prudente. Partimos del hecho de que nos parece incuestionable la existencia de interacción entre ámbitos, concretamente entre los de tiempo libre, trabajo de producción y trabajo de reproducción y de que no es realista plantear posiciones extremas. Precisamente lo que nos interesa investigar es la naturaleza de esta relación: su contenido, en qué planos opera más intensamente (el material, el de las actitudes, el intencional y el simbólico), el sentido de dicha relación así como los agentes y soportes sociales sobre los que se asienta. La conceptualización de dicha articulación es lo que denominaremos «vida cotidiana». Ésta nos parece las opción más enriquecedora.

Sobre la base de las anteriores consideraciones, tres son los objetivos que nos planteamos en la investigación:

1. En primer lugar, analizar cómo se configura y articula la vida cotidiana, tanto en sus aspectos más objetivables como en las representaciones y elementos simbólicos. Se trata de ver cómo se constituyen en cada uno de los ámbitos diferentes agrupaciones (tipos) coherentes de actividades y de representaciones y, a partir de ellas, cómo se configuran tipologías de relación entre los ámbitos. En otras palabras, se trata de elaborar una tipología que dé cuenta de cómo las personas articulan diariamente trabajo productivo, trabajo reproductivo y tiempo libre. Ello significa cómo se entrelaza lo individual - autonomíacon lo estructural (contexto), que tiene siempre algo de construcción.

Por un lado podemos pensar en tipos fundamentales o puros - cada uno construido prioritariamente sobre uno de los tres ámbitos- y por otro estaremos abiertos a encontrar tipos en transición o mixtos. Al final trataremos de llegar a una tipología única de interacción entre los ámbitos, es decir, que sea resultado de diversas combinaciones de los tres. Es aquí, y en los tipos mixtos, donde podremos esperar lo resultados más originales de los cambios que puedan operarse en la vida cotidiana de las personas.

2. Como segundo objetivo nos planteamos ir más allá y no quedarnos al nivel de tipologías de ámbitos y de interacción, aunque éstas se sitúen en los tres niveles: un primero más objetivista y los otros más intencionales y simbólicos. Pretendemos llegar a los aspectos dinámicos de la vida cotidiana a partir de la comprensión de cómo y por qué se configuran unas u otras formas de vida cotidiana, fundamentalmente a partir de la captación de los grupos sociales que están presentes en la tipología de articulación. Es importante también descubrir, dentro de este objetivo, las contradicciones que puedan aparecer en la articulación que realizan los diferentes grupos. Estos grupos social es aparece- 
rán caracterizados por indicadores sociales y por representaciones, pero también, y es lo que nos interesa particularmente, por las estructuras de sus ámbitos u orientaciones de sentido. Las contradicciones (o correspondencias) que puedan darse no serán solamente debidas al mestizaje posible entre tipos puros, sino también al trasvase o transición de incoherecias (o coherencias) que se encuentren entre los distintos niveles: una inscripción manifiesta en un tipo en la primera instancia y en otro, no coherente con el primero, a nivel actitudinal o simbólico, por ejemplo.

Este objetivo es muy importante para entender los cambios en la vida cotidiana y su evolución, las posibilidades que ciertas personas tienen de llevar a cabo vidas cotidianas diversas de las que ahora tienen, o para diseñar políticas que vayan dirigidas a mejorar la vida cotidiana de determinados colectivos.

3. En tercer lugar, y como elemento más globalizador, nos planteamos un obje tivo teórico importante en el planteamiento: buscar las claves explicativas teóricas que den cuenta de la interacción de los tres ámbitos - trabajo productivo, reproductivo y tiempo libre- y de la dinámica y grupos sociales correspondientes que vayan más allá - es nuestro supuesto- de la exclusiva division que introduce el mercado. Si estas claves teóricas existen, permitirían entender mejor las relaciones entre trabajo y sociedad, entender más a fondo la dinámica de la división sexual del trabajo, las relaciones entre aspiraciones a una mayor disponibilidad del tiempo libre, la trasmutación que se opera de los valores del trabajo, etc. Por supuesto, todo ello posibilitaría un acercamiento teórico mucho más sólido al análisis de la vida cotidiana como marco en el que se inscribe esta interacción entre los ámbitos.

\section{Marco teórico de la investigación}

\subsection{El marco de cada uno de los ámbitos}

Una parte muy importante de las consideraciones que, como punto de partida, hemos realizado, son, evidentemente, patrimonio común de muchas otras investigaciones. Por eso, de ellas hemos asumido ciertos conceptos y elaboraciones, de las que vamos a dar cuenta:

- Las transformaciones del trabajo productivo han sido explicadas básicamente por el desarrollo de la teoría marxista o bien de la teoría funcionalista del capital humano y de las profesiones, lo que ha permitido entender las desigualdades en el trabajo, aś como las relaciones de poder en el mismo.

- Respecto al trabajo reproductivo, éste puede ser estudiado con la teoría del patriarcado y del modelo producción/reproducción; lo cual nos permite entender la relación entre género y los diferentes trabajos que realizan las personas: productivo y reproductivo.

- Las actividades y transformaciones que se dan en el ámbito de las actividades de tiempo libre (o de no trabajo), se han estudiado a través de la teo- 
ría marxista como mero tiempo de recuperación y también a través de una visión funcionalista. Y últimamente a partir de una perspectiva propia otorgándole un carácter más autónomo y creativo.

\subsection{La necesaria vinculación teórica entre los ámbitos}

No es posible estudiar, a partir únicamente de estas teorías, la vida cotidiana como articulación de los tres ámbitos que hemos señalado, ni - y más importante todavía - encontrar una respuesta satisfactoria al estudio de las transformaciones de cada ámbito con sus propias herramientas teóricas. La originalidad de esta investigación está precisamente en concatenar los aspectos teóricos parciales, válidos en cada ámbito, en vistas a ofrecer una perspectiva más global que dé cuenta de la articulación que en la realidad se da entre ellos. Veamos brevemente al gunos aspectos de nuestra propuesta teórica.

- En la sociedad moderna el trabajo-profesión (trabajo productor de bienes y servicios) organiza y estructura no sólo la actividad de las personas en torno al mismo, sino también aspectos importantes del resto de la vida, mucho más allá de la diferenciación que dan las posiciones de asal ariado y propietario en la producción. Entendemos esta estructuración u organización en términos de poder e influencia («estructuración social» de Giddens), y con carácter desigual: tal organización no se da uniformemente, puesto que en el trabajo existen oportunidades diversas («dualización» de Piore y Sabel, «segmentación» de G ordon, 1986).

Esto es así en un doble sentido. Las personas pueden acceder a uno u otro trabajo en función de su posición fuera del mismo (origen social, estudios, posición en la familia, ofertas que hacen las empresas). El tener uno u otro puesto de trabajo hace variar ostensiblemente su poder y su capacidad negociadora. Por tanto, mantenemos que hay una clara influencia del trabajo-profesión sobre otros ámbitos de la vida, pero diferenciadamente. Sin embargo, no hay una influencia unidireccional; bien al contrario, Io que se es y se hace en los ámbitos externos al trabajo influye decisivamente en el trabajo (O ffe).

- Para entender en su profundidad el significado del trabajo es necesario ir más allá de la lógica productiva, asumiendo que se trata de una realidad más global que se liga tanto a la producción de bienes y servicios como a la reproducción de la vida («producción de productores», Benería); es más, hay que asumir que el trabajo reproductivo es imprescindible para que la producción funcione y que, por tanto, la actividad que se realiza en ésta no puede ser entendida sin tener en cuenta la que tiene lugar en aquélla. Pero justamente la subordinación de un importante colectivo humano, las muje res, respecto al trabajo productivo, proviene de la rígida división sexual del trabajo en su conjunto en dos realidades diferenciadas: el trabajo productivo y el trabajo reproductivo. 
H asta aquí las aportaciones teóricas que nos pueden permitir trazar el puente entre trabajo productivo y trabajo reproductivo. Pero nuestros intereses van más allá, se refieren a la organización de la vida cotidiana como marco social que da sentido a la articulación entre dichos ámbitos. Las proposiciones que configuran nuestra propuesta teórica son las siguientes:

- La segmentación en el trabajo explica no sólo las diversas posiciones de poder dentro de ese ámbito, sino que contribuye a explicar la posición de poder-subordinación en el ámbito del trabajo reproductivo y en el tiempo libre (no trabajo) (tener-no tener, tiempo de no trabajo pasivo-tiempo de no trabajo activo). La razón está en que la capacidad negociadora diferenciada según segmentos es causa y efecto de las posiciones sociales de las personas.

- El grado y la intensidad de la división sexual del trabajo global contribuye notablemente a explicar no sólo diversas posiciones en el trabajo reproductivo, sino también en los otros dos ámbitos señalados. Pero también incide notablemente en la socialización de valores, símbolos y ethos que trasladan esas posiciones desiguales de hombres y mujeres al ámbito del trabajo y al del tiempo de no trabajo.

- La vivencia del tiempo libre (no trabajo) con una mayor o menor autonomía (los valores de autorrealización fuera del trabajo, la importancia de la individualidad en la configuración de la persona social) contribuye a configurar una división sexual del trabajo diversa y una accesibilidad diferenciada al trabajo y a la posición en el mismo.

\subsection{El modelo teórico de vida cotidiana}

a) Concepciones sobre la vida cotidiana. ¿Sociología o sociologías de la vida cotidiana?

0 tro de los elementos teóricos clave en la investigación se refiere al concepto mismo de «vida cotidiana». La sociología de la vida cotidiana no está caracte rizada precisamente por ser una teoría unificada, sino más bien como una teoría paraguas u omnibus de reenganche de diversas procedencias: hay muchas disciplinas que se reclaman de la sociología de la vida cotidiana. Entonces ila sociología de la vida cotidiana es un mosaico, es una conjunción de fragmentos mal ensamblados, es un cuerpo disciplinario? ¿H ay al go que pueda caracterizarla de manera específica? Patricia A. Adler, Peter Adler y Andrea Fontana (1987) distinguen, con todo, entre dicho cuerpo relativamente desarticulado en que consiste la sociología de la vida cotidiana, al gunos principios mayores que le dan alguna identidad:

- Primero, la crítica a la macrosociología. Las teorías que originan o se reclaman de la vida cotidiana provienen de una desafección por todo lo macrosociológico, sobre todo en la sociología norteamericana de los años cincuenta. Los estudios macro son considerados y criticados por los distintos autores 
y tendencias adscribibles a la sociología de la vida cotidiana como positivistas, tendentes a la absolutización y globalización de los hechos sociales, así como a su naturalización, alejados del individuo y de la dialéctica y/o dualismo que se establece entre sujeto y objeto, alejados también de la interacción y de la situación social en la que transcurre su vida diaria, incapacitados para captar la complejidad del mundo de cada día.

- La segunda idea compartida consiste en tomar en cuenta el contexto situa cional en el análisis de los fenómenos sociales, tratando de respetar la integridad de dicho fenómeno y estudiando a las personas en su medio natural social cotidiano.

- El tercer aspecto es el modelo de actor que preconizan a partir de la idea de interacción. Como realidad interior al individuo, se trata de un modelo de actor basado sobre todo en las construcciones simbólicas a las que las personas llegan por la socialización en la interacción. Como realidad exterior, la visión que se deriva es que la estructura y la interacción no existen fuera de las personas que interactúan. Estas corrientes o teorías parten de dos filosofías en la consideración de lo micro. Una de ellas, tomando a M ead como origen y a partir de una visión pragmática del comportamiento, evoluciona hacia el interaccionismo simbólico de Blumer o hacia la dramaturgia de $\mathrm{G}$ offman. 0 tras, basadas en la fenomenología y a través de la fenomenología sociológica de Schutz, toman diversas líneas de pensamiento, por ejemplo: la etnometodología de G arfinkel, el constructivismo de Berger y Luckmann.

Los años setenta y ochenta aportan una nueva generación de sociólogos de la vida cotidiana, pero siempre en el marco de una supuesta unidad de la disciplina y una diversidad de perspectivas vinculadas a las precedentes. $N$ uevas orientaciones se han añadido a las precedentes. Por ejemplo, lo que se podría llamar la sociología existencial, dentro de una visión más teórica, la sociología de las emociones, más sustantiva, y el análisis conversacional, más metodológico; añadiríamos la introducción de tema de la vida cotidiana en corrientes neomarxistas, Lefebvre, $\mathrm{H}$ eller.

b) El modelo de vida cotidiana aquí adoptado

Estando básicamente de acuerdo con las grandes líneas con las que se ha caracterizado precedentemente la o las sociologías de la vida cotidiana, la visión y el modelo de vida cotidiana que proponemos en esta investigación enfatiza y se corresponde con una visión más integradora entre una orientación microsociológica y macrosociológica, entre una perspectiva más estructural y otra más centrada en el actor social, en el análisis de la situación y de la interacción.

No estaremos de acuerdo con una perspectiva que considerara únicamente la vida cotidiana como la esfera de la privacidad, equiparándola al dominio de la individualidad o al de las estrategias de resolución de la problemática y de la intendencia de cada día, sin que se atisbe en ella la mínima incidencia de los condicionamientos sociales de más envergadura. 
Esta visión tiene algo que ver con otra, que tampoco compartimos, que convierte la vida cotidiana en la parte irrelevante y residual de todo lo que es social, equiparando lo social sólo a lo que es institucional, organizacional, estructural o global y, extensivamente, a lo histórico e «importante». Ésta es la idea que ha dominado en la mayor parte de las teorías macrosociológicas cuando hacen alguna incursión en la vida cotidiana.

También nuestra concepción se distancia de las que consideran la vida cotidiana como el único referente real y como germen primario de toda la vida social, como si toda la realidad social fuera una prolongación o extensión conceptual de la vida cotidiana, sin ruptura de continuidad desde los aspectos más microsociológicos hasta los más estructurales e institucionales. Es una perspectiva que corresponde en buena medida a las concepciones clásicas de la vida cotidiana que hemos enumerado anteriormente.

En fin, también nos alejamos de una visión que contempla la vida cotidiana como una especie de núcleo irreductible e impenetrable a lo social, a manera de entidad autoconsciente, inerte e invariante con relación a la dinámica de las realidades sociales más globales, tal como se deja entender de las perspectivas de $M$ affesoli, C atani, C erteau.

H oy día aparecen nuevas teorías sociológicas generales que tienden a ser más integradoras - Alexander, C ollins, Knorr-C etina, Cicourel, Bourdieu, Giddens, Touraine, Lefebvre- , tratando de evitar enfoques exclusivamente objetivistas o subjetivistas. Tienden a incorporar una visión de un actor que entiende, comprende y actúa sobre las estructuras estando a su vez coaccionado por ellas. En líneas generales, nuestro modelo va en una dirección integradora e interaccionista, considerando los hechos sociales, las estructuras sociales y las construcciones simbólicas, producidas o reproducidas en las situaciones cotidianas a partir de la interacción entre actores; ellos mismos, a su vez, producto y resultado de la interacción y de las determinaciones del contexto estructural. En este sentido, nos encontramos dentro de las corrientes integradoras mencionadas y en la de otros pensadores que han trabajado de una manera más específica sobre la temática de la vida cotidiana: Bertaux, Lalive d'Epinay, Ferrarotti, H eller, Javeau, Lefebvre, Remy.

$O$ tro de los el ementos importantes integrado en nuestro modelo de la vida cotidiana es su carácter articulador. El modelo de vida cotidiana se entiende en esta investigación como el marco conceptual y teórico de una realidad social compleja que va más allá de la división que de los hechos sociales establecen las sociologías particulares o especializadas. En esta investigación, la vida cotidiana será considerada como una realidad social que corresponde a la articulación einteracción que se realiza entre el ámbito del trabajo productor de bienes y servicios, TP; el ámbito de las actividades que producen y reproducen la persona humana, TR, y, en fin, el ámbito de las actividades del tiempo libre, TL. Se entiende, además, que dicha articulación de ámbitos no se realiza sólo en el nivel factual y objetivable - actividades y prácticas- , sino también, y es uno de los elementos interesantes de nuestra investigación, tanto en los niveles de las representaciones y actitudes como en el de los elementos simbólicos y estruc- 
turas de sentido. Esto se traduce, por ejemplo, en cómo organiza cada individuo o grupo su tiempo para hacer frente a las lógicas que vienen de la producción, de la reproducción y del tiempo libre; en cómo se mantiene la coherencia, o no, de la personalidad social en la interferencia entre los distintos niveles, actividades, representaciones y procesos intencionales; en cómo se vinculan los objetivos en cada campo con el resto de los campos o ámbitos; en cómo se transmutan los sentidos y unos ámbitos son referentes para explicar los otros; en cómo funcionan los valores simbólicos en cada ámbito y entre sí; en cómo se vinculan las relaciones primarias y secundarias; etc. La vida social de las personas y de los grupos es única y, salvo un inexplicable autismo social en cada grupo y ámbito, constituye un todo articulado en la realidad social del día a día, o de otras temporalidades que se considerasen. $M$ anteniendo cada ámbito una lógica y coherencia, la vida cotidiana consistirá en la articulación que se produce en el transcurso y flujo de las diversas situaciones de día a día entre dichos ámbitos e instancias. El conjunto según las distintas temporalidades consideradas produce un continuo coherente, o incoherente, pero con sentido.

Así pues, el contenido de la vida cotidiana estará dado por diversos componentes que se han de tener en cuenta en este intento de definición. 1) Primero, los ámbitos o referentes puestos en juego en las situaciones 0 sucesión de situaciones anal izables: TP, TR y TL. Pueden estar definidos previamente, como es nuestro caso, o pueden ser descubiertos en el proceso de aná lisis. 2) Segundo, las situaciones o sucesión de situaciones y su correspondiente encadenamiento. No habrá en nuestra investigación un análisis de situaciones en «directo» sino indirecto, dado que la memoria de los investigados reproduce situaciones, o partes, pasadas o posibles. 3) Tercero, el núcleo analizable en una situación, o sucesión de situaciones, está constituido por varios elementos: unos son más objetivables y manifiestos (actividades y prácticas); otros son más intencionales (representaciones y estados intencionales); otros son más actitudinales (predisponiendo a determinadas prácticas), y, en fin, otros elementos son más simbólicos (ethos, valores, etc.) y dan los ámbitos de significación y sentido. En nuestra investigación hemos tenido en cuenta todos los elementos en los diferentes niveles. Estos componentes y elementos se vinculan entre sí, ofreciendo unas tipologías de articulación. A partir de ellas se pueden diferenciar distintos grupos sociales, agentes 0 actores, que les corresponden. Ese aspecto del análisis es capital, pues es el que más implica e incide en la naturaleza social de la vida cotidiana, ya que nos da los diferentes grupos sociales que son los sujetos de diferentes modos de vida cotidiana $y$, en consecuencia, las posibilidades de cambio o de inercia social en este nivel.

En principio, los modos de vida no están en absoluto contextualizados directamente por variables Ilamadas «estructurales»: edad, género, categoría socioeconómica, ingresos, etc. No es evidente, aunque podemos trabajar con este supuesto, que se haya de dar una estrecha correlación entre la caracterización de los modos de vida cotidiana y los grupos formados por las categorías sociales clásicas. Pero no podemos ignorar, con todo, el potente peso estructurador 
que sobre el conjunto de la vida de la gente poseen las variables contextuales mencionadas. No obstante, también es verdad que es quizás en la vida cotidiana donde se puede manifestar con más vigor, aunque quizás con más clandestinidad, la capacidad de resistencia o de innovación de las personas con relación a los grupos hegemónicos y dominantes de los factores sociales más estructurales.

\section{Los niveles de la aproximación metodológica a los ámbitos y a su interacción}

Sobre la base de este marco teórico nos hemos planteado tres niveles de aproximación a nuestros objetivos.

\subsection{Primer nivel}

En el primer nivel hemos analizado la articulación objetivable de la vida cotidiana, sintetizándola en los tres ámbitos que estamos tomando como punto de análisis (TP, TR y TL). D os son los aspectos a explicar: por un lado, encontrar tipologías de articulación principalmente sobre la base de los tiempos de dedicación; por otro, captar los factores que condicionan dicha articulación (edad, género, origen social, estudios, etc.). N o se trata de una mera descripción, sino de buscar las razones explicativas de la mencionada tipología. Para ello, volviendo al marco teórico, avanzamos unos posibles ejes explicativos que convendrá explorar.

- En primer lugar nos referiremos a la caracterización básica del trabajo (TP) hoy en las sociedades desarrolladas. El modelo de la segmentación del trabajo puede explicar que no todas las personas tengan las mismas oportunidades de trabajo y que no todos los trabajos ofrezcan las mismas posibilidades. Pero además señalará que esas oportunidades diferenciadas tienen mucho que ver con lo que socialmente se puede dar fuera del trabajo. Consideramos este nivel explicativo como punto de partida, puesto que pensamos que las personas están aún hoy en día muy condicionadas por lo que son o no son (o son directamente o por referencia - al padre, al marido, etc. - ) respecto al trabajo, a la profesión, a la ocupación en el ámbito del mercado de trabajo.

- En segundo lugar hay que referirse al modelo producción/reproducción que pretende explicar la situación de las personas en ambos ámbitos a la vez, el productivo y el reproductivo, es decir, se está o no en el trabajo productivo o bien se está de una u otra manera en relación con lo que se es en el ámbito reproductivo; en el grado en que están comprometidos en lo reproductivo, las personas están o no en el ámbito productivo (hacer no quiere decir ser, depender de, etc.), o están de diversas maneras.

- Por último nos aproximamos a la explicación de la articulación del trabajo productivo y del trabajo reproductivo (y del tiempo libre) desde la perspectiva de la vida cotidiana, es decir, desde la perspectiva de la trayectoria 
de vida que la gente ha desarrollado y que explica la ubicación de las personas en uno u otro de los tres ámbitos o en los mestizajes de ámbitos. Ello con relación a determinadas actividades, espacios, relaciones, en incluso valoraciones.

Los datos para realizar este análisis son extraídos de una amplia encuesta existente sobre condiciones de vida y hábitos de la población en la región metropolitana de Barcelona. Son datos recogidos previamente al diseño de esta investigación y, por tanto, algunas de las limitaciones de nuestro análisis son explicables por este origen de los datos.

\subsection{Segundo nivel}

El segundo nivel nos lleva a analizar, dentro del proceso de socialización que está en la base de la articulación material de la vida cotidiana tal como la analizamos en el nivel anterior, las percepciones sobre el trabajo productivo, el trabajo reproductivo y el tiempo libre en las personas estudiadas. En cierto modo también es un estudio de las ideologías que sustentan la vida cotidiana, de lo que la legitima ante los demás y, en cierta medida, ante nosotros mismos. Los resultados de este nivel nos permitirán ver el grado de «coherencia» 0 «incoherencia» que existe entre la materialidad de la vida cotidiana de las personas y los deseos, las aspiraciones y proyecciones que realizan y, de esta manera, reformar, rehacer o confirmar las tipologías iniciales.

En este caso los datos provienen de una encuesta sobre actitudes y percepciones, realizada ya específicamente para esta investigación, que diseña los tres ámbitos sobre los que se analiza la vida cotidiana.

\subsection{Tercer nivel}

En este nivel hemos querido llegar más al fondo de las estructuras de sentido de la vida cotidiana, analizando la relación y coherencia (o incoherencia) entre los tipos de vida previamente establecidos y las estructuras de sentido. Ello es lo que establecerá la consistencia (o la inconsistencia) definitiva de los tipos de articulación y de los grupos sociales encontrados. Ambas, consistencia y/o inconsistencia, serán objeto de las explicaciones adecuadas. En particular nos interesa saber hasta qué punto estos dominios simbólicos o estructuras de sentido se corresponden, por un lado, con factores clásicos explicativos: posición social sobre todo en las categorías socioeconómicas y socioprofesionales, edad, sexo, etc. y, por otro, con los tipos que provienen y se configuran en cada uno de los ámbitos tomados en sí mismos: estratificación y segmentación del mercado del trabajo, doble presencia y su articulación. Ello implica comprender en qué grado las personas, cuya vida cotidiana estudiamos, se sienten y actúan como protagonistas, qué grado de autonomía o heteronomía, libertad o subordinación hay en sus vidas cotidianas con relación a los determinismos sociales clásicos. 
En este caso los datos son resultado de entrevistas en profundidad con personas que responden a las características de las tipologías dibujadas en el primer nivel estudiado.

\section{Bibliografía}

Adler, P.A.; Adler, P.; Fontana, A. (1987). «Everyday life sociology». Ann. Rev. of Sociology, núm. 13.

AgUILAR, S. y otros. Els contextos de l'acció sindical a Espanya. Barcelona: Fundació Jaume Bofill.

Alòs-M oner, R.; M IgUÉLez, F.; ReCio, A. (1988). El trabajo precario en Cataluña. La industria textil en el Vallès O ccidental. Barcelona: CERES-CO N C.

- (1989). Relaciones laborales y trabajo precario en el comercio. Barcelona: CERESCONC.

Carrasco, C.; Borderías, C.; Alem any, C. (1994). El trabajo y las mujeres. Barcelona: Fuhem-Icaria.

CAST ELL, M . y otros (1986). El desafío tecnológico. España y las nuevas tecnologías. M adrid: Alianza Editorial.

CASTILLO, J.J. (comp.) (1988). Lasnuevas formas de organización del trabajo. M adrid: MTSS.

GoRd ON , D .; EdwARDS, R.; REICH, M . (1986). Trabajo segmentado, trabajadores divididos. M adrid: M TSS.

H OM S, O y otros (1988). «C ambios en la empresa y requerimientos de cualificación en España». Revista Sociología del Trabajo, núm. 2. Siglo XXI.

KERN, H .; SCH UM AN N, M . (1988). El fin de la división del trabajo. M adrid: M TSS. LOPE, A. (1994). Innovació tecnològica. Barcelona: Fundació Jaume Bofill.

M ART ín ARTILES, A. (1995). Flexibilidad y relaciones laborales. Estrategias empresariales y acción sindical. M adrid: Consejo Económico y Social.

M IGUÉLEZ, F.; Ló PEZ, P. (1988). «El trabajo en la pequeña empresa española». Papers, núm. 32.

M in ISterio de Trabajo y Seguridad So cial (1995). La política de empleo en España. $M$ adrid: Colección Informes y Estudios.

O fFE, Claus (1992). La sociedad del trabajo. M adrid: Alianza Editorial.

Papel es de Economía Española (1985). M onografía, núm. 22. M adrid.

PIORE, M.; SABEL, Ch. (1990). La segunda ruptura industrial. M adrid: Alianza Editorial.

SANCh Ís, E. (1984). El trabajo a domicilio en el País Valenciano. M adrid: Instituto de la Mujer.

Varios Auto Res (1985). Enquesta M etropolitana de Barcelona. Barcelona: IEM B.

- (1990). Enquesta M etropolitana de Barcelona. Barcelona: IEM B. 